\title{
Clinical Characteristics of Osimertinib Responder in Non-Small Cell Lung Cancer Patients with EGFR-T790M Mutation
}

\author{
Akihiro Yoshimura ${ }^{1}$ D , Tadaaki Yamada ${ }^{1, * \mathbb{D}}$, Naoko Okura ${ }^{1}$, Takayuki Takeda ${ }^{2} \mathbb{D}$, \\ Kazuki Hirose ${ }^{3}$, Yutaka Kubota ${ }^{3}$, Shinsuke Shiotsu ${ }^{4}$, Osamu Hiranuma ${ }^{5}$, Yusuke Chihara ${ }^{1}$, \\ Nobuyo Tamiya ${ }^{1}$, Yoshiko Kaneko ${ }^{1}$, Junji Uchino ${ }^{1}$ (D) and Koichi Takayama ${ }^{1}$ \\ 1 Department of Pulmonary Medicine, Graduate School of Medical Science, Kyoto Prefectural University of \\ Medicine, 465 Kajii-cho, Kamigyo-ku, Kyoto 602-8566, Japan; aki-y@koto.kpu-m.ac.jp (A.Y.); \\ ku-n07@koto.kpu-m.ac.jp (N.O.); c1981311@koto.kpu-m.ac.jp (Y.C.); koma@koto.kpu-m.ac.jp (N.T.); \\ kaneko-y@koto.kpu-m.ac.jp (Y.K.); uchino@koto.kpu-m.ac.jp (J.U.); takayama@koto.kpu-m.ac.jp (K.T.) \\ 2 Department of Respiratory Medicine Uji-Tokushukai Medical Center, Kyoto 611-0041, Japan; \\ dyckw344@yahoo.co.jp \\ 3 Department of Respiratory Medicine, Japanese Red Cross Kyoto Daini Hospital, Kyoto 602-8026, Japan; \\ k-hirose09@outlook.jp (K.H.); ytkkbt@yahoo.co.jp (Y.K.) \\ 4 Department of Respiratory Medicine, Japanese Red Cross Kyoto Daiichi Hospital, Kyoto 605-0981, Japan; \\ sshiotsu@gmail.com \\ 5 Department of Respiratory Medicine, Otsu City Hospital, Otsu 520-0804, Japan; osamu319@true.ocn.ne.jp \\ * Correspondence: tayamada@koto.kpu-m.ac.jp; Tel.: +81-75-251-5513; Fax: +81-75-251-5376
}

Received: 20 February 2019; Accepted: 13 March 2019; Published: 15 March 2019

\begin{abstract}
Osimertinib is a mutant-selective EGFR inhibitor that is effective against non-small cell lung cancer (NSCLC) in patients with the EGFR-T790M mutation, who are resistant to EGFR-tyrosine kinase inhibitors (EGFR-TKIs). However, the factors affecting response to osimertinib treatment are unknown. In this retrospective study, 27 NSCLC patients with the EGFR-T790M mutation were enrolled at five institutions in Japan. Among several parameters tested, the progression-free survival (PFS) associated with the initial EGFR-TKIs was positively correlated with the PFS after osimertinib treatment $(p=0.021)$. The median PFS following osimertinib treatment and the overall survival (OS) were longer in patients who responded to osimertinib than in those who did not (17.7 months versus 3.5 months, $p=0.009$ and 24.2 months versus 13.5 months, $p=0.021$, respectively). A multivariate analysis demonstrated that the PFS with initial EGFR-TKIs was significantly related to the PFS with osimertinib treatment $(p=0.035)$, whereas osimertinib response was significantly related to the PFS and OS with osimertinib treatment ( $p=0.016$ and $p=0.006$, respectively). Our retrospective observations indicate that PFS following the initial EGFR-TKI treatment and the response rate to osimertinib might be promising predictors for effective osimertinib treatment in NSCLC patients with the EGFR-T790M mutation.
\end{abstract}

Keywords: osimertinib; EGFR-T790M mutation; non-small cell lung cancer; biomarker; retrospective study

\section{Introduction}

The development of molecular-targeted therapy has markedly improved clinical outcomes in non-small cell lung cancer (NSCLC) patients with alterations in the driver genes. NSCLC patients with activating epidermal growth factor receptor (EGFR) mutations, such as exon 19 deletion and exon 21 point mutation (L858R), respond significantly better to first- and second-generation EGFR-tyrosine 
kinase inhibitors (EGFR-TKIs) than to platinum-based chemotherapy [1,2]. However, almost all patients acquire resistance to initial EGFR-TKIs in approximately 10-12 months. Several acquired resistance mechanisms to initial EGFR-TKIs are known, including gatekeeper mutations such as EGFR-T790M, activation of bypass signaling, epithelial mesenchymal transition and transformation to small-cell lung cancer [3,4]. The EGFR-T790M mutation is the most common acquired resistance mechanism to first- and second-generation EGFR-TKIs [3,5]. Phase III clinical trials have revealed that the third-generation EGFR-TKI osimertinib provides a better progression-free survival (PFS) than platinum-based chemotherapy in NSCLC patients who have EGFR-T790M [6]. Therefore, osimertinib has been approved in the United States, Japan, and other countries for cases with EGFR-mutated NSCLC harboring EGFR-T790M mutations and acquired resistance to initial EGFR-TKIs, including gefitinib, erlotinib, and afatinib. More recently, osimertinib was approved as the first-line of treatment for advanced EGFR-mutated NSCLC patients based on the results of a phase III clinical trial [7].

Although osimertinib is effective in most NSCLC patients with the EGFR-T790M mutation, a 71\% objective response rate and 10.1 months median PFS were recorded in a clinical trial; however, the mechanisms underlying patient response to osimertinib treatment are still unclear [6]. Ariyasu et al. reported that an increased expression of the T790M allele product is among the several EGFR-activating mutations that have been predicted to be involved in the response to osimertinib using droplet digital polymerase chain reaction analyses [8]. However, further investigations are warranted to reveal biomarkers that can more conveniently and cost-effectively detect responders to osimertinib among NSCLC patients with EGFR-T790M mutations because $6 \%$ of them showed the disease progression treated with osimertinib in a clinical trial [6].

In this retrospective study, we investigated predictive clinical biomarkers associated with osimertinib efficacy based on the profiles of NSCLC patients with the EGFR-T790M mutation after acquiring initial resistance to EGFR-TKIs.

\section{Results}

\subsection{Patient Characteristics}

A total of 78 EGFR-mutant NSCLC patients underwent re-biopsy to detect EGFR-T790M mutation after acquiring resistance to the initial EGFR-TKIs. After excluding 51 patients who met the exclusion criteria, 27 patients with EGFR-T790M mutations were finally enrolled (Figure S1). Patient characteristics after detection of EGFR-T790M mutations are summarized in Table 1. The median age was 73 years (range, $44-84)$; 18 patients $(66.7 \%)$ were female; 20 patients $(74.1 \%)$ were nonsmokers; and the majority of patients (92.6\%) indicated a Performance Status (PS) of 0 and 1 . The most prevalent history of disease included the incidence of adenocarcinoma (96.3\%), 10 patients (37.0\%) had relapse after surgery, and all the patients had exon 19 deletion or an L858R deletion in exon 21; these were the most common mutation types in EGFR. Twenty-four patients $(88.9 \%)$ responded to the initial EGFR-TKIs. EGFR-T790M mutation was detected in five patients (18.5\%) through liquid biopsy, in whom tumor re-biopsy was not conducted. Our results showed all of the EGFR-T790M mutation was detected in tumors after acquiring resistance to EGFR-TKIs, but not in tumors at a baseline, which indicated that it was caused by acquired resistance.

Table 1. Patients baseline characteristics.

\begin{tabular}{ccc}
\hline \multicolumn{2}{c}{ Patients' Characteristics } & $N=\mathbf{2 7}, \boldsymbol{n}, \mathbf{( \% )}$ \\
\hline \multirow{2}{*}{ Age } & Median (Range) & $78.0(47.0-88.0)$ \\
\multirow{2}{*}{ Sex } & Male & $9(33.3)$ \\
& Female & $18(66.7)$ \\
\hline \multirow{2}{*}{ PS } & 0,1 & $25(92.6)$ \\
& 2 & $2(7.4)$ \\
\hline \multirow{2}{*}{ Histology } & Adenocarcinoma & $26(96.3)$ \\
& Squamous cell carcinoma & $1(3.7)$ \\
\hline
\end{tabular}


Table 1. Cont.

\begin{tabular}{ccc}
\hline \multicolumn{2}{c}{ Patients' Characteristics } & $\boldsymbol{N}=\mathbf{2 7}, \boldsymbol{n ,}(\mathbf{\%})$ \\
\hline \multirow{3}{*}{ Smoking status } & Never-smoker & $20(74.1)$ \\
& Ever-smoker & $4(14.8)$ \\
& Current-smoker & $3(11.1)$ \\
\hline \multirow{2}{*}{ Stage } & III & $4(14.8)$ \\
& IV & $13(48.1)$ \\
& Postoperative recurrence & $10(37.0)$ \\
\hline \multirow{2}{*}{ EGFR mutation status } & Exon 19 deletion & $20(74.1)$ \\
& Exon 21 L858R mutation & $7(25.9)$ \\
\hline \multirow{2}{*}{ Initial EGFR-TKI } & Gefitinib & $17(63.0)$ \\
& Erlotinib & $6(22.2)$ \\
Initial TKI response & Afatinib & $4(14.8)$ \\
& CR, PR & $24(88.9)$ \\
& SD, PD & $3(11.1)$ \\
\hline
\end{tabular}

Abbreviations: PS, performance status; EGFR, epidermal growth factor receptor; EGFR-TKI, EGFR-tyrosine kinase inhibitor; TKI, tyrosine kinase inhibitor; $\mathrm{CR}$, complete response; PR, pertial response; $\mathrm{SD}$, stable diseaase; $\mathrm{PD}$, progressive disease; $N$, number.

\subsection{Predictor Factors in Osimertinib Treatment}

To evaluate the clinical factors to predict the efficacy of osimertinib treatment in NSCLC patients with EGFR-T790M mutations, we first categorized two groups: patients in whom the PFS with osimertinib treatment was more than 8 months were classified into the "long PFS group," and patients in whom the duration was less than 8 months were classified into the "short PFS group". Of the 27 patients with EGFR-T790M mutations, 17 patients $(63.0 \%)$ belonged to the long PFS group and 10 patients $(37.0 \%)$ belonged to the short PFS group. There was no significant difference in patient profiles of the two groups as shown in Table 2.

Table 2. Patients baseline characteristics classified by progression-free survival (PFS) duration of osimertinib.

\begin{tabular}{|c|c|c|c|c|}
\hline \multicolumn{2}{|c|}{ Patients' Characteristics } & $\begin{array}{c}\text { PFS Duration of } \\
\text { Osimertinib } \geq 8 \text { Months }\end{array}$ & $\begin{array}{c}\begin{array}{c}\text { PFS Duration of } \\
\text { Osimertinib }<8 \text { Months }\end{array} \\
N=10 \\
n(\%)\end{array}$ & $p$ Value \\
\hline & & & & \\
\hline Age & Median (Range) & $78.0(49.0-88.0)$ & $71.0(47.0-83.0)$ & 0.247 \\
\hline \multirow{2}{*}{ Sex } & Male & $5(29.4)$ & $4(40.0)$ & 0.683 \\
\hline & Female & $12(70.6)$ & $6(60.0)$ & \\
\hline \multirow{2}{*}{ PS } & 0,1 & $16(94.1)$ & $9(90.0)$ & 1 \\
\hline & 2 & $1(5.9)$ & $1(10.0)$ & \\
\hline \multirow{2}{*}{ Histology } & Adenocarcinoma & $17(100.0)$ & $9(90.0)$ & 0.37 \\
\hline & Squamous cell carcinoma & $0(0.0)$ & $1(10.0)$ & \\
\hline \multirow{2}{*}{ Smoking status } & Never-smoker & $12(70.6)$ & $8(80.0)$ & 0.678 \\
\hline & Smoker & $5(29.4)$ & $2(20.0)$ & \\
\hline \multirow{3}{*}{ Stage } & III & $2(11.8)$ & $2(20.0)$ & 0.472 \\
\hline & IV & $7(41.2)$ & $6(60.0)$ & \\
\hline & Postoperative recurrence & $8(47.1)$ & $2(20.0)$ & \\
\hline \multirow{2}{*}{ EGFR mutation status } & Exon 19 deletion & $12(70.6)$ & $8(80.0)$ & 0.678 \\
\hline & Exon 21 L858R mutation & $5(29.4)$ & $2(20.0)$ & \\
\hline \multirow{3}{*}{ EGFR-TKI } & Gefitinib & $11(64.7)$ & $6(60.0)$ & 0.195 \\
\hline & Erlotinib & $5(29.4)$ & $1(10.0)$ & \\
\hline & Afatinib & $1(5.9)$ & $3(30.0)$ & \\
\hline \multirow{3}{*}{ Re-biopsy site } & Intrathoracic & $12(70.6)$ & $5(50.0)$ & 0.623 \\
\hline & Extrathoracic & $2(11.8)$ & $3(30.0)$ & \\
\hline & Liquid & $3(17.6)$ & $2(20.0)$ & \\
\hline
\end{tabular}


We next examined the difference in treatment-related factors in both groups and the results are presented in Table 3. The response rate to osimertinib was significantly higher in patients from the long PFS group than in those from the short PFS group $(88.2 \%$ versus $40.0 \%, p=0.025)$. The rate of longer PFS with initial EGFR-TKIs (more than 8 months) tended to be higher in patients in the long PFS group than in those in the short PFS group ( $<8$ months) $(88.2 \%$ versus $50.0 \%, p=0.065)$. Therefore, we focused on the two clinical parameters, PFS with initial EGFR-TKI treatment and the response rate to osimertinib, as treatment-related factors for osimertinib.

Table 3. Patients' characteristics of clinical course classified progression-free survival duration of osimertinib.

\begin{tabular}{|c|c|c|c|c|}
\hline \multicolumn{2}{|c|}{ Patients' Characteristics } & $\begin{array}{c}\begin{array}{c}\text { PFS Duration of } \\
\text { Osimertinib } \geq 8 \\
\text { Months }\end{array} \\
N=17\end{array}$ & $\begin{array}{c}\begin{array}{c}\text { PFS Duration of } \\
\text { Osimertinib }<8 \\
\text { Months }\end{array} \\
N=10 \\
n(\%)\end{array}$ & $p$ Value \\
\hline \multirow{2}{*}{ PFS duration of initial TKI } & $\geq 8$ months & $15(88.2)$ & $8(80.0)$ & 0.065 \\
\hline & $<8$ months & $2(11.8)$ & $2(20.0)$ & \\
\hline \multirow{2}{*}{ Osimertinib response } & $\mathrm{CR} / \mathrm{PR}$ & $15(88.2)$ & $4(40.0)$ & 0.025 \\
\hline & $\mathrm{SD} / \mathrm{PD}$ & $2(11.8)$ & $6(60.0)$ & \\
\hline Osimertinib shrinkage & Median (Range) & $50.0(13.5-100.0)$ & $20.6(-126.4-66.5)$ & 0.006 \\
\hline \multirow{2}{*}{$\begin{array}{l}\text { Platinum doublet therapy } \\
\text { after osimertinib }\end{array}$} & + & $0(0.0)$ & $3(30.0)$ & 0.041 \\
\hline & - & $17(100.0)$ & $7(70.0)$ & \\
\hline
\end{tabular}

Of these parameters, median PFS with osimertinib was 17.7 months in the long PFS group (95\% confidence interval [CI] 9.0-22.0 months) and was 3.2 months in the short PFS group (95\% CI 1.2-9.6 months) ( $p=0.021)$. Meanwhile, there was no significant difference in OS between the two groups ( $p=0.337$ ) (Figure 1A,B). Median PFS with osimertinib was 17.7 months in osimertinib responders (CR/PR) (95\% CI: 9.0 months-not evaluable [NE]) and 3.5 months in osimertinib non-responders (SD/PD) (95\% CI: 0.3 months $-\mathrm{NE})(p=0.009)$. In addition, the osimertinib responders had a longer OS than the non-responders (24.2 months [95\% CI: 22.1 months-NE] and 13.5 months [95\% CI: 0.3 months-NE], $p=0.021$ ) although patients of the short PFS group showed a significantly better opportunity for undergoing platinum doublet therapy after acquiring resistance to osimertinib than patients with longer PFS with osimertinib $(p=0.041)$ (Figure 1C,D).

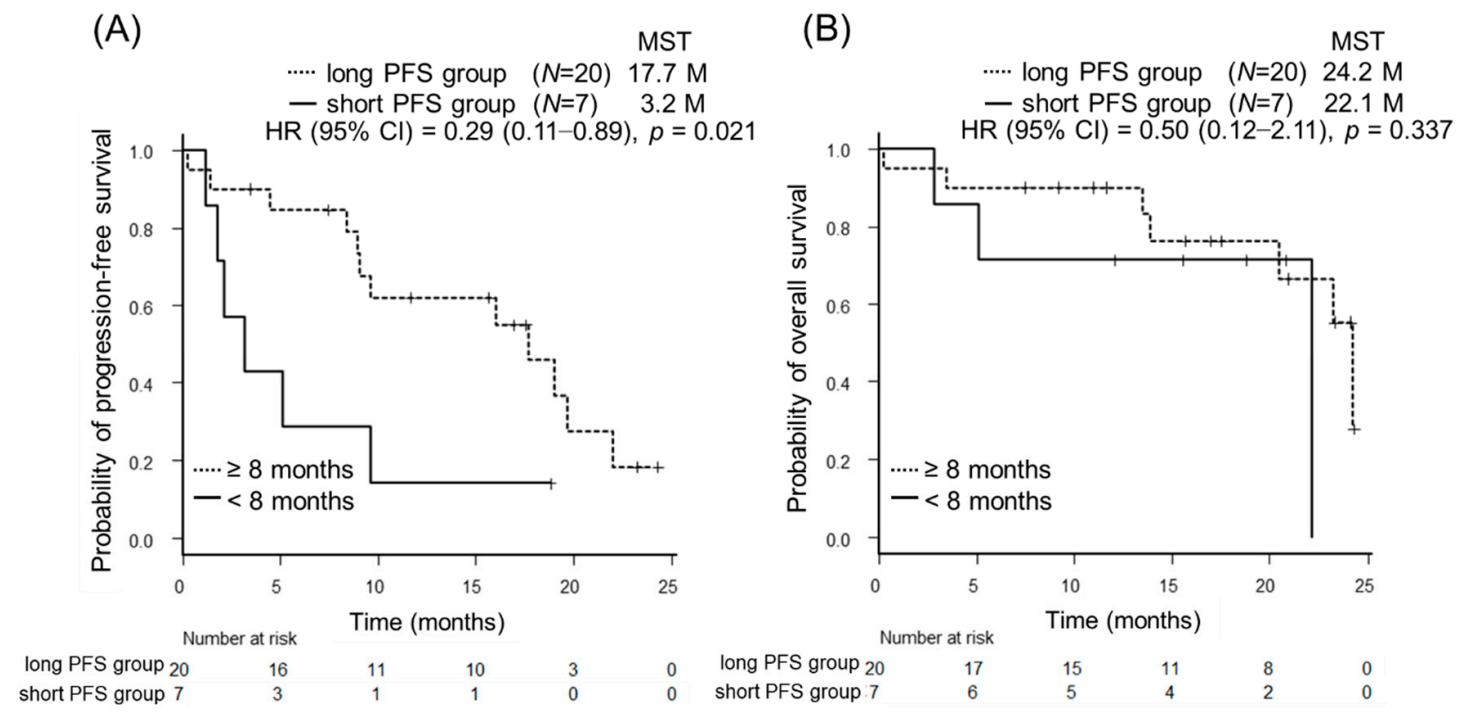

Figure 1. Cont. 

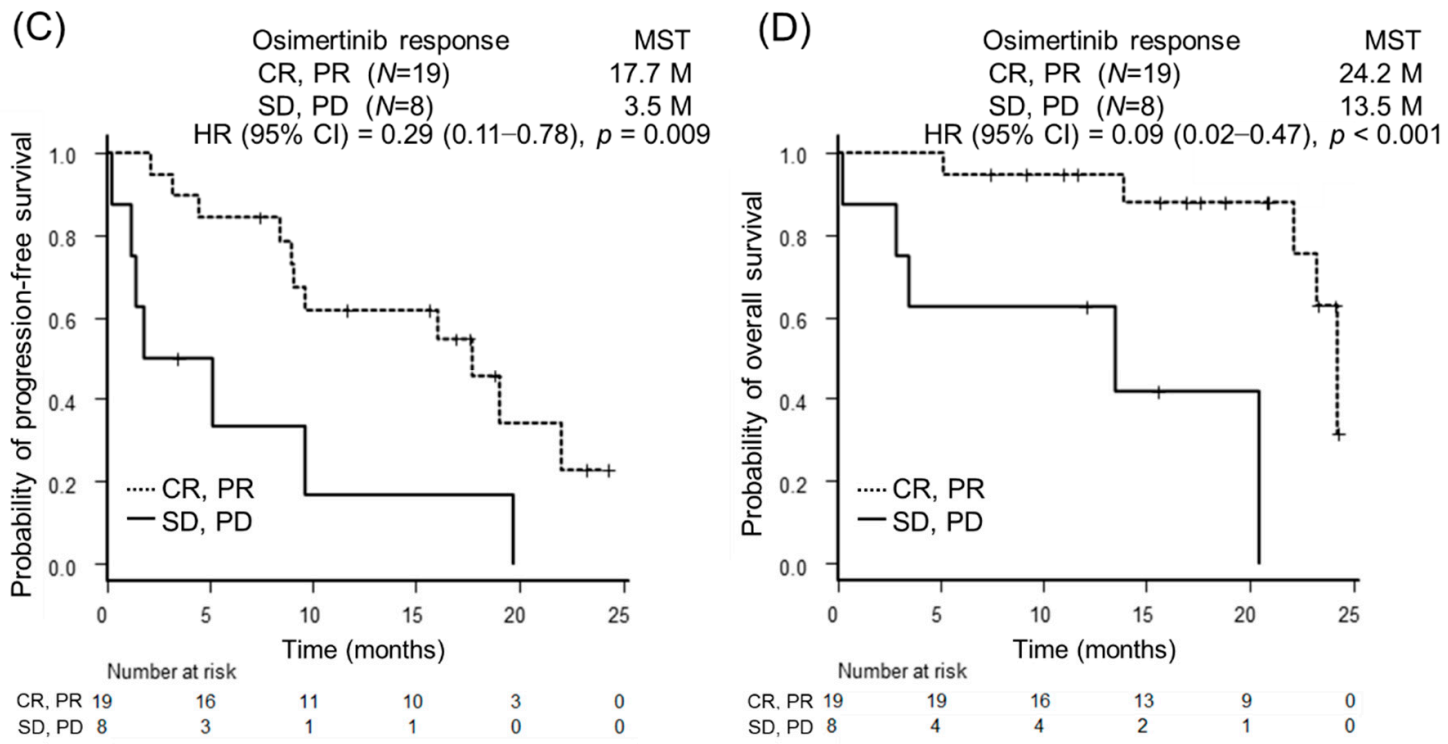

Figure 1. Kaplan-Meier survival curves for progression free survival (PFS) and overall survival (OS) for the PFS duration of initial EGFR-TKI treatment and osimertinib response. (A,B) PFS and OS of the EGFR-T790M-mutant non-small cell lung cancer (NSCLC) patients with the long PFS for the initial EGFR-TKI $(N=20)$ or those with the short PFS $(N=7)$. The median PFS was significantly longer in patients with the long PFS for the initial EGFR-TKI than in those with the short PFS (17.7 months versus 3.2 months, $p=0.021)$. There was no significant difference in OS between the two groups ( $p=0.337)$. (C,D) PFS and OS of EGFR-T790M-mutant NSCLC patients with osimertinib responsiveness (CR/PR) $(N=19)$ or those with osimertinib non-responsiveness $(\mathrm{SD} / \mathrm{PD})(N=8)$. The median PFS and OS were significantly longer in osimertinib responders than in osimertinib non-responders (17.7 months versus 3.5 months, $p=0.009)$, (24.2 months versus 13.5 months, $p=0.021)$, respectively.

The multivariate analysis demonstrated that PFS with the initial EGFR-TKIs was significantly related to the PFS with osimertinib (HR 0.31, 95\% CI $=0.11-0.92, p=0.035$ ), whereas osimertinib response was significantly related to the PFS with osimertinib and the OS (HR $0.29,95 \% \mathrm{CI}=0.11-0.80$, $p=0.016$; HR 0.09, 95\% CI $=0.02-0.50, p=0.006$, respectively) (Table 4).

Given that the response rate with osimertinib was significantly correlated with the PFS, we further examined the response rate relative to the baseline according to exposure to osimertinib. The median maximum tumor shrinkage (MTS) of the group with longer PFS and short PFS were $50.0 \%$ and $20.6 \%$, respectively, which indicated a significant association between PFS and osimertinib treatment $(p=0.006)$ (Figure 2A-C). 
Table 4. Univariate and multivariate analysis of patients' characteristics and the clinical course.

\begin{tabular}{|c|c|c|c|c|c|c|c|c|}
\hline \multirow{3}{*}{ Variables } & \multicolumn{4}{|c|}{ Progression-Free Survival } & \multicolumn{4}{|c|}{ Overall Survival } \\
\hline & \multicolumn{2}{|c|}{ Univariate Analysis } & \multicolumn{2}{|c|}{ Multivariate Analysis } & \multicolumn{2}{|c|}{ Univariate Analysis } & \multicolumn{2}{|c|}{ Multivariate Analysis } \\
\hline & HR, Mean $(95 \%$ CI $)$ & $p$ Value & HR, Mean $(95 \%$ CI $)$ & $p$ Value & HR, Mean $(95 \%$ CI $)$ & $p$ Value & HR, Mean $(95 \%$ CI) & $p$ Value \\
\hline $\begin{array}{c}\text { Age at detection of T790M }(<75 / \geq 75 \\
\text { years })\end{array}$ & $1.38(0.53-3.57)$ & 0.504 & & & $0.71(0.20-2.55)$ & 0.603 & & \\
\hline Performance status $(0-1 / 2)$ & $0.27(0.06-1.26)$ & 0.073 & & & $\mathrm{NE}$ & 0.557 & & \\
\hline $\begin{array}{l}\text { Disease stage (postoperative recurrence/ } \\
\text { stage III or IV) }\end{array}$ & $0.31(0.1-0.95)$ & 0.03 & $0.41(0.12-1.41)$ & 0.16 & $0.30(0.06-1.41)$ & 0.105 & & \\
\hline $\begin{array}{l}\text { EGFR status (exon19 deletion/exon21 } \\
\text { L858R mutation) }\end{array}$ & $0.84(0.45-1.58)$ & 0.177 & & & $1.53(0.31-7.42)$ & 0.597 & & \\
\hline EGFR-TKI (afatinib/gefitinib, erlotinib) & $3.01(0.81-11.16)$ & 0.084 & & & $4.05(0.96-17)$ & 0.039 & $8.15(1.10-60.38)$ & 0.04 \\
\hline $\begin{array}{l}\text { Initial EGFR-TKI response (CR, PR/SD, } \\
\text { PD) }\end{array}$ & $0.63(0.14-2.83)$ & 0.543 & & & $4.89(0.61-39.16)$ & 0.099 & & \\
\hline $\begin{array}{l}\text { Initial EGFR-TKI PFS (more than } 8 \\
\text { months/less than } 8 \text { months) }\end{array}$ & $0.31(0.11-0.89)$ & 0.021 & $0.30(0.10-0.90)$ & 0.031 & $0.50(0.12-2.11)$ & 0.337 & & \\
\hline Re-biopsy (tissue/liquid) & $0.62(0.17-2.31)$ & 0.476 & & & NE & 0.319 & & \\
\hline Osimetinib response (CR, PR/SD, PD) & $0.29(0.11-0.78)$ & 0.009 & $0.44(0.15-1.33)$ & 0.15 & $0.09(0.02-0.47)$ & $<0.001$ & $0.02(0.00-0.27)$ & 0.002 \\
\hline
\end{tabular}


(A)

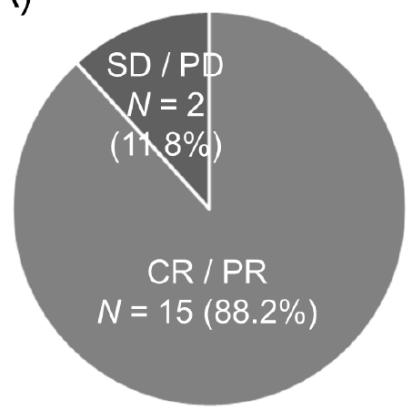

$\mathrm{CR} / \mathrm{PR}=\mathrm{SD} / \mathrm{PD}$
(B)

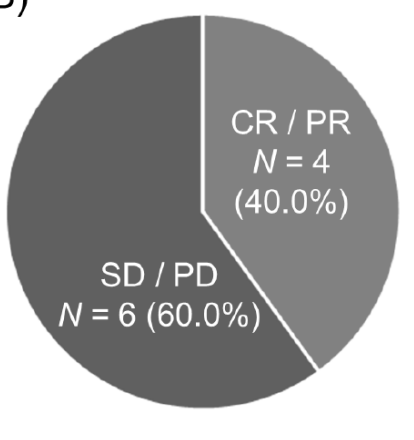

$\mathrm{CR} / \mathrm{PR}=\mathrm{SD} / \mathrm{PD}$

(C)

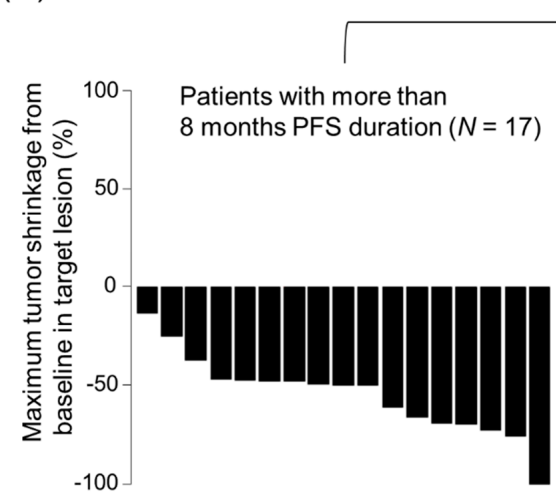

$p=0.006$

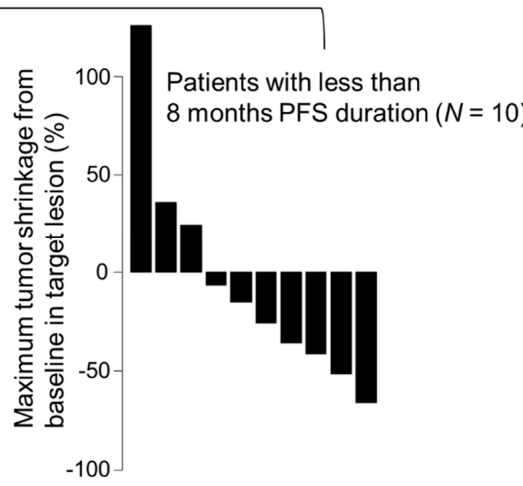

(D)

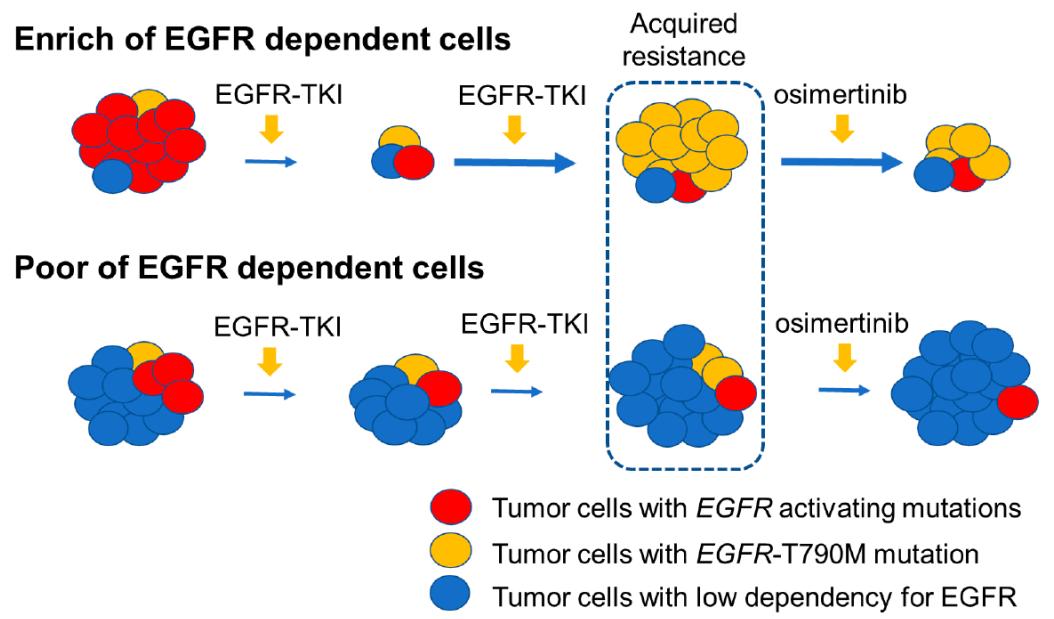

Figure 2. The osimertinib response of NSCLC patients with EGFR-T790M mutation treated with osimertinib on the PFS duration of osimertinib. (A) Frequency of the best overall response to osimertinib treatment among EGFR-T790M mutant NSCLC patients with the more than 8 months PFS duration $(N=17)$. (B) Frequency of the best overall response to osimertinib treatment among EGFR-T790M-mutant NSCLC patients with the less than 8 months PFS duration $(N=10)$. (C) The median maximum tumor shrinkage rate relative to baseline in 27 NSCLC patients with EGFR-T790M mutation treated with osimertinib. The PFS duration of 17 patients was more than 8 months and that of 10 patients was less than 8 months. The median maximum tumor shrinkage rate in these patients indicated a significant association with the osimertinib response $(50.0 \%$ and $20.6 \%$, respectively, $p=0.006$ ). (D) Schematic diagram showing that tumors with resistance to EGFR-TKIs may be heterogeneous, consisting of both EGFR signal dependency with EGFR activating mutation (red) or with EGFR-T790M mutation (yellow), and EGFR signal independency (blue) populations. 


\section{Discussion}

The EGFR-T790M mutation was detected in approximately $50 \%$ of the patients, who had acquired resistance to first- and second-generation EGFR-TKIs [3,5]. We revealed that the response rate to initial EGFR-TKI administration positively correlates with the detection of T790M mutation in EGFR mutated NSCLC patients [9]. Moreover, this present study showed that initial EGFR-TKI response is a useful predictor for osimertinib treatment in NSCLC patients with the EGFR-T790M mutation. This finding is meaningful from the point of view of the detection of responders during initial EGFR-TKI treatment.

Osimertinib is known as a mutant-selective EGFR inhibitor that is effective for EGFR-T790M-positive NSCLC after acquired resistance to initial EGFR-TKIs [10,11]. There was a significant difference in the median PFS with osimertinib treatment between EGFR-T790M-positive and -negative NSCLC patients after acquiring resistance to initial EGFR-TKIs [12]. Wang S. et al. showed that patients with acquired EGFR-T790M mutation showed a higher frequency of exon 19 deletion in EGFR, shorter PFS with osimertinib, and prolonged OS than those with primary EGFR-T790M mutation [13]. In this study, all patients gained EGFR-T790M mutations from the acquired resistance phase, and there was no difference between 20 in exon 19 deletion in EGFR and 7 in exon 21 L858R mutation in EGFR with regard to clinical outcomes, such as PFS with osimertinib and OS.

However, such patients with EGFR-T790M-mutant NSCLC were expected to have other diverse resistance mechanisms that indicate the various responses to osimertinib because of the increase in intratumor heterogeneity during initial EGFR-TKI treatment. Indeed, the high tumor mutation burden in patients with EGFR-mutant NSCLC was involved in poor prognosis of EGFR-TKIs treatment [14]. Therefore, the dependency on EGFR signaling at the baseline might be critical for predicting osimertinib response after acquiring resistance to initial EGFR-TKIs in NSCLC patients with the EGFR-T790M mutation (Figure 2D).

Previous studies have shown that first-line EGFR-TKI responders have significantly longer PFS than the non-responders among EGFR mutant NSCLC patients [15]. The proportion of tumor cells that respond to osimertinib might be expected to correlate with the longer PFS associated with osimertinib treatment, in consistency with the initial EGFR-TKIs. Moreover, the population of EGFR-T790M mutation before osimertinib treatment might correlate with the dependency on EGFR signaling, which may lead to the prediction of the effectiveness of the osimertinib treatment (Figure 2D).

Several mechanisms were reported for the acquired resistance to osimertinib in EGFR-T790M mutated NSCLC patients, including EGFR-C797S mutation, bypass signal activation, and transformation to small cell lung cancer [16-18]. To overcome these resistance mechanisms, combined therapies, such as combination with anti-angiogenesis inhibitors, are ongoing in multiple clinical trials. In addition, preclinical studies have showed that combination with osimertinib may be promising for EGFR-mutant NSCLC patients with or without T790M mutations $[19,20]$. The NSCLC patients with EGFR-T790M mutation after acquiring resistance to osimertinib had significantly longer PFS in osimertinib treatment than those without EGFR-T790M mutation [21]. Interestingly, patients that did not have EGFR-T790M mutation induced EGFR-independent mechanisms on acquiring resistance, such as activation of bypass signaling and transformation to small cell lung cancer. Our observations indicate a positive correlation between the response to osimertinib and the PFS with osimertinib treatment. These findings suggest that the population of EGFR-dependent tumor cells at the time of pretreatment with osimertinib may have an impact on the PFS following osimertinib treatment (Figure 2D).

The emergence of EGFR-T790M mutation is reported as a good prognosis factor, when the patient acquires resistance to initial EGFR-TKIs [22]. A previous study demonstrated that high expression of AXL in pre-treatment tumors was relatively related to poor outcomes of EGFR-TKI treatment, including osimertinib [20]. Our observations also revealed that the response rate of osimertinib has a significant relationship with survival. Therefore, patients with longer PFS with osimertinib treatment are expected to maintain the tumors with EGFR-T790M mutation after acquiring resistance to osimertinib; this 
observation suggests that PFS might be involved in survival. However, further studies are required to validate this point.

This study has several limitations, which are as follows: First, it has a small sample size and was retrospective in nature. However, the median PFS of all the patients in this study was similar to that observed in a phase III study (9.6 months [95\% CI: 5.1-19.7] vs. 10.1 months [95\% CI: 8.3-12.3]) [6]. Second, this study was based on a Japanese cohort of patients only. Third, we had various biases on patient conditions when EGFR-TKIs were started, even though the study was performed in multiple centers and the timing of evaluation by CT scanning was in the following 1-3 months. Therefore, further prospective study is warranted to identify the role of longer PFS duration of the initial EGFR-TKIs administration on the osimertinib response in EGFR-T790M-positive NSCLC.

\section{Materials and Methods}

\subsection{Patients}

We retrospectively enrolled 39 patients with advanced or postoperative recurrent EGFR-mutant NSCLC; re-biopsy samples were obtained from these patients. The samples were either from tumors or the plasma after resistance was acquired to the initial EGFR-TKIs. The patients were enrolled at five institutions in Japan between May 2014 and January 2018. The exclusion criteria were as follows: (1) no osimertinib treatment, (2) discontinuation of initial EGFR-TKI treatment owing to adverse events, and (3) no measurable lesions.

All patients were evaluated for imaging responses, including complete response (CR), partial response (PR), stable disease (SD), and progressive disease (PD), by conventional CT scanning according to the instructions of RECIST version 1.1. We obtained patients' clinical data from medical records retrospectively; the information included age, sex, smoking status, Eastern Cooperative Oncology Group Performance Status (PS), histological subtype, clinical stage, EGFR mutation status, initial EGFR-TKI administered, initial EGFR-TKI response, PFS with initial EGFR-TKI, re-biopsy site, history of platinum-based chemotherapy after acquiring resistance to osimertinib, PFS with osimertinib, and overall survival (OS). We set the initial EGFR-TKI PFS cutoff to eight months because the median PFS with gefitinib and erlotinib were reported as approximately eight months in phase 3 clinical trial [23]. PFS was defined as the period from osimertinib treatment initiation to disease progression by RECIST or the period till 30 June, 2018. OS was defined as the period from osimertinib treatment initiation to death or until 30 June, 2018. This study protocol was approved by the Ethics Committees (Kyoto Prefectural University of Medicine) of each hospital on 08 February 2018 (ethic code: ERB-C-1107). The TNM stage was classified using version 7 of the TNM stage classification system.

\subsection{EGFR Mutation Analysis}

EGFR mutations were detected using the polymerase chain reaction method for tumor and plasma samples by sequencing exons 18-21; the sequencing was performed at commercial clinical laboratories: SRL, Inc. and BML, Inc. (Tokyo, Japan).

\subsection{Statistical Analysis}

The Cox proportional-hazard model, which accounted for several factors of the patient profiles was used. To analyze the PFS, times to events were estimated using the Kaplan-Meier method and compared by the log-rank test. The PFS was censored at the date of disease progression. Predictive factors for osimertinib response to EGFR-T790M mutation in NSCLC patients were identified using univariate and multivariate logistic analyses. All statistical analyses were performed using EZR for Windows, version 1.35 (Saitama Medical Center, Jichi Medical University, Saitama, Japan). $p$ values less than 0.05 indicated statistical significance. 


\section{Conclusions}

Our retrospective observations suggest that the PFS with initial EGFR-TKI treatment and the response rate to osimertinib might be promising predictors for osimertinib treatment in patients with EGFR-T790M-positive NSCLC; this may be due to the ratio of tumor heterogeneity that might be enriched during initial EGFR-TKI treatment. Further experiments are needed to validate these observations.

Supplementary Materials: The following are available online at http://www.mdpi.com/2072-6694/11/3/ 365/s1, Figure S1: Patient flowchart of NSCLC with EGFR-T790M mutation after they acquired resistance to initial EGFR-TKIs.

Author Contributions: Conceptualization, T.Y. and K.T.; methodology, A.Y.; formal analysis, A.Y.; investigation, N.O., Y.C., N.T., Y.K. (Yoshiko Kaneko) and J.U.; resources, A.Y., T.T., K.H., Y.K. (Yutaka Kubota), S.S., O.H.; data curation, T.Y.; writing—original draft preparation, A.Y.; writing—review and editing, T.Y.; supervision, K.T.

Funding: This research received no external funding.

Acknowledgments: We thank the patients, their families, and all investigators involved in this study. We are also grateful to Rumi Makino and Hiroko Tamaru for assisting with the administrative work.

Conflicts of Interest: All authors have no conflict of interest to declare.

\section{References}

1. Maemondo, M.; Inoue, A.; Kobayashi, K.; Sugawara, S.; Oizumi, S.; Isobe, H.; Gemmam, A.; Harada, M.; Yoshizawa, H.; Kinoshita, I.; et al. Gefitinib or chemotherapy for non-small-cell lung cancer with mutated EGFR. N. Engl. J. Med. 2010, 362, 2380-2388. [CrossRef] [PubMed]

2. Sequist, L.V.; Yang, J.C.; Yamamoto, N.; O’Byrne, K.; Hirsh, V.; Mok, T.; Geater, S.L.; Orlov, S.; Tsai, C.M.; Boyer, M.; et al. Phase III study of afatinib or cisplatin plus pemetrexed in patients with metastatic lung adenocarcinoma with EGFR mutations. J. Clin. Oncol. 2013, 31, 3327-3334. [CrossRef] [PubMed]

3. Sequist, L.V.; Waltman, B.A.; Dias-Santagata, D.; Digumarthy, S.; Turke, A.B.; Fidias, P.; Bergethon, K.; Shaw, A.T.; Gettinger, S.; Cosper, A.K.; et al. Genotypic and histological evolution of lung cancers acquiring resistance to EGFR inhibitors. Sci. Transl Med. 2011, 3, 75ra26. [CrossRef] [PubMed]

4. Yano, S.; Takeuchi, S.; Nakagawa, T.; Yamada, T. Ligand-triggered resistance to molecular targeted drugs in lung cancer: Roles of hepatocyte growth factor and epidermal growth factor receptor ligands. Cancer Sci. 2012, 103, 1189-1194. [CrossRef] [PubMed]

5. Wu, S.G.; Liu, Y.N.; Tsai, M.F.; Chang, Y.L.; Yu, C.J.; Yang, P.C.; Yang, J.C.H.; Wen, Y.F.; Shih, J.Y. The mechanism of acquired resistance to irreversible EGFR tyrosine kinase inhibitor-afatinib in lung adenocarcinoma patients. Oncotarget 2016, 7, 12404-12413. [CrossRef] [PubMed]

6. Mok, T.S.; Wu, Y.L.; Ahn, M.J.; Garassino, M.C.; Kim, H.R.; Ramalingam, S.S.; Shepherd, F.A.; He, Y.; Akamatsu, H.; Theelen, W.S.M.E.; et al. Osimertinib or Platinum-Pemetrexed in EGFR T790M-Positive Lung Cancer. N. Engl. J. Med. 2017, 376, 629-640. [CrossRef] [PubMed]

7. Soria, J.C.; Ohe, Y.; Vansteenkiste, J.; Reungwetwattana, T.; Chewaskulyong, B.; Lee, K.H.; Dechaphunkul, A.; Imamura, F.; Nogami, N.; Kurata, T.; et al. Osimertinib in Untreated EGFR-Mutated Advanced Non-Small-Cell Lung Cancer. N. Engl. J. Med. 2018, 378, 113-125. [CrossRef] [PubMed]

8. Ariyasu, R.; Nishikawa, S.; Uchibori, K.; Oh-Hara, T.; Yoshizawa, T.; Dotsu, Y.; Koyama, J.; Saikia, M.; Sonodaa, T.; Kitazonoa, S.; et al. High ratio of T790M to EGFR activating mutations correlate with the osimertinib response in non-small-cell lung cancer. Lung Cancer 2018, 117, 1-6. [CrossRef] [PubMed]

9. Yoshimura, A.; Yamada, T.; Okura, N.; Takeda, T.; Furutani, W.; Kubota, Y.; Shiotsu, S.; Hiranuma, O.; Nishioka, N.; Chihara, Y.; et al. The impact of the tumor shrinkage by initial EGFR inhibitors according to the detection of EGFR-T790M mutation in patients with non-small cell lung cancer harboring EGFR mutations. BMC Cancer 2018, 18, 1241. [CrossRef] [PubMed]

10. Kobayashi, Y.; Mitsudomi, T. Not all epidermal growth factor receptor mutations in lung cancer are created equal: Perspectives for individualized treatment strategy. Cancer Sci. 2016, 107, 1179-1186. [CrossRef] [PubMed] 
11. Kohsaka, S.; Nagano, M.; Ueno, T.; Suehara, Y.; Hayashi, T.; Shimada, N.; Takahashi, K.; Suzuki, K.; Takamochi, K.; Takahashi, F.; et al. A method of high-throughput functional evaluation of EGFR gene variants of unknown significance in cancer. Sci. Transl. Med. 2017, 9, eaan6566. [CrossRef] [PubMed]

12. Janne, P.A.; Yang, J.C.; Kim, D.W.; Planchard, D.; Ohe, Y.; Ramalingam, S.S.; Ahn, M.J.; Kim, S.W.; Su, W.C.; Horn, L.; et al. AZD9291 in EGFR inhibitor-resistant non-small-cell lung cancer. N. Engl. J. Med. 2015, 372, 1689-1699. [CrossRef] [PubMed]

13. Wang, S.; Yan, B.; Zhang, Y.; Xu, J.; Qiao, R.; Dong, Y.; Zhang, B.; Zhao, Y.; Zhang, L.; Qian, J.; et al. Different characteristics and survival in non-small cell lung cancer patients with primary and acquired EGFR T790M mutation. Int. J. Cancer 2018. [CrossRef] [PubMed]

14. Offin, M.; Rizvi, H.; Tenet, M.; Ni, A.; Sanchez-Vega, F.; Li, B.T.; Drilon, A.; Kris, M.G.; Rudin, C.M.; Schultz, N.; et al. Tumor Mutation Burden and Efficacy of EGFR-Tyrosine Kinase Inhibitors in Patients with EGFR-Mutant Lung Cancers. Clin. Cancer Res. 2018, 25, 1063-1069. [CrossRef] [PubMed]

15. Wu, T.H.; Hsiue, E.H.; Lee, J.H.; Lin, C.C.; Liao, W.Y.; Ho, C.C.; Shih, J.Y.; Yu, C.J.; Yang, C.H. Best Response According to RECIST During First-line EGFR-TKI Treatment Predicts Survival in EGFR Mutation-positive Non-Small-cell Lung Cancer Patients. Clin. Lung Cancer 2018, 19, e361-e372. [CrossRef] [PubMed]

16. Thress, K.S.; Paweletz, C.P.; Felip, E.; Cho, B.C.; Stetson, D.; Dougherty, B.; Lai, Z.; Markovets, A.; Vivancos, A.; Kuang, Y.; et al. Acquired EGFR C797S mutation mediates resistance to AZD9291 in non-small cell lung cancer harboring EGFR T790M. Nat Med. 2015, 21, 560-562. [CrossRef] [PubMed]

17. Minari, R.; Bordi, P.; Tiseo, M. Third-generation epidermal growth factor receptor-tyrosine kinase inhibitors in T790M-positive non-small cell lung cancer: Review on emerged mechanisms of resistance. Trans. Lung Cancer Res. 2016, 5, 695-708. [CrossRef] [PubMed]

18. Yang, Z.; Yang, N.; Ou, Q.; Xiang, Y.; Jiang, T.; Wu, X.; Bao, H.; Tong, X.; Wang, X.; Shao, Y.W.; et al. Investigating Novel Resistance Mechanisms to Third-Generation EGFR Tyrosine Kinase Inhibitor Osimertinib in Non-Small Cell Lung Cancer Patients. Clin. Cancer Res. 2018, 24, 3097-3107. [CrossRef] [PubMed]

19. Akamatsu, H.; Koh, Y.; Ozawa, Y.; Fujimoto, D.; Hata, A.; Katakami, N.; Tomii, K.; Shimokawa, T.; Yamamoto, N. Osimertinib With Ramucirumab in EGFR-mutated, T790M-positive Patients With Progression During EGFR-TKI Therapy: Phase Ib Study. Clin. Lung Cancer 2018, 19, e871-e874. [CrossRef] [PubMed]

20. Taniguchi, H.; Yamada, T.; Wang, R.; Tanimura, K.; Adachi, Y.; Nishiyama, A.; Tanimoto, A.; Takeuchi, S.; Araujo, L.H.; Boroni, M.; et al. AXL confers intrinsic resistance to osimertinib and advances the emergence of tolerant cells. Nat. Commun. 2019, 10, 259. [CrossRef] [PubMed]

21. Oxnard, G.R.; Hu, Y.; Mileham, K.F.; Husain, H.; Costa, D.B.; Tracy, P.; Feeney, N.; Sholl, L.M.; Dahlberg, S.E.; Redig, A.J.; et al. Assessment of Resistance Mechanisms and Clinical Implications in Patients With EGFR T790M-Positive Lung Cancer and Acquired Resistance to Osimertinib. JAMA Oncology. 2018, 4, 1527-1537. [CrossRef] [PubMed]

22. Ma, G.; Zhang, J.; Jiang, H.; Zhang, N.; Yin, L.; Li, W.; Zhou, Q. Epidermal growth factor receptor T790M mutation as a prognostic factor in EGFR-mutant non-small cell lung cancer patients that acquired resistance to EGFR tyrosine kinase inhibitors. Oncotarget 2017, 8, 99429-99437. [CrossRef] [PubMed]

23. Urata, Y.; Katakami, N.; Morita, S.; Kaji, R.; Yoshioka, H.; Seto, T.; Iwamoto, Y.; Kanehara, M.; Fujimoto, D.; Ikeda, N.; et al. Randomized Phase III Study Comparing Gefitinib With Erlotinib in Patients With Previously Treated Advanced Lung Adenocarcinoma: WJOG 5108L. J. Clin. Oncol. 2016, 34, 3248-3257. [CrossRef] [PubMed]

(C) 2019 by the authors. Licensee MDPI, Basel, Switzerland. This article is an open access article distributed under the terms and conditions of the Creative Commons Attribution (CC BY) license (http:/ / creativecommons.org/licenses/by/4.0/). 